INPLASY

PROTOCOL

To cite: Zhang et al. Efficacy, safety, and retention rate of extended-release divalproex versus conventional delayedrelease divalproex: A protocol for systematic review and meta-analysis. Inplasy protocol 2021110090. doi:

10.37766/inplasy2021.11.0090

Received: 24 November 2021

Published: 24 November 2021

Corresponding author:

Hongbin Sun

shb001369x@163.com

Author Affiliation:

Chengdu BOE Hospital

Support: Grant no. 2016C001.

Review Stage at time of this

submission: Data extraction.

Conflicts of interest:

None declared.

\section{Efficacy, safety, and retention rate of extended-release divalproex versus conventional delayed-release divalproex: A protocol for systematic review and meta-analysis}

\author{
Zhang, CQ1; Bai, XY2; Wan, Y3; Li, HY4; Sun, HB55.
}

Review question / Objective: We aim to systematically examine and compare the efficacy, safety and retention rate of ER divalproex (VPA-ER) and conventional DR divalproex (VPA-DR) on patients with epilepsy.

Condition being studied: To our knowledge, comparison and conversion studies about VPA-ER and conventional VPA-DR are lacked. Small-sample studies analyzing the safety and efficacy of VPA-ER in different populations remain controversial. Therefore, this study aims to systematically review the safety, efficacy and retention rate of VPA-ER and VPA-DR by meta-analysis.

INPLASY registration number: This protocol was registered with the International Platform of Registered Systematic Review and Meta-Analysis Protocols (INPLASY) on 24 November 2021 and was last updated on 24 November 2021 (registration number INPLASY2021110090).

\section{INTRODUCTION}

Review question / Objective: We aim to systematically examine and compare the efficacy, safety and retention rate of ER divalproex (VPA-ER) and conventional DR divalproex (VPA-DR) on patients with epilepsy.

Rationale: Valproic acid and its derivatives, including valproate sodium (sodium valproate), divalproex and divalproex sodium are all known as valproate (VPA).VPA is the most commonly applied first-generation broad-spectrum antiepileptic drug (AED) to generalized and focal epilepsies in children and adults approved by the Food and Drug Administration (FDA).VPA has been approved in several formulations, including the original delayedrelease tablet (e.g., Depakote), enteric-coated particles, 
sprinkle capsules, sustainedrelease (e.g., Depakine Chrono) and a more recently approved, extended-release tablet (e.g., Depakote ER). In ER formulations, the dosing interval is usually extended to minimize the dosing frequency . In addition, they can potentially minimize the spikes in the maximum plasma concentrations (Cmax) at a steady-state and maintain the relatively constant or flat plasma drug concentration. Moreover, they can minimize concentration-related AEs. Oncedaily VPA-ER is featured by the hydrophilic polymer matrix controlled-release tablet system, which allows for the slow release of drugs in the stomach, small intestine, and large intestine for 18-24 h . Compared with the standard twice-daily (BID) DR formulation, once-daily VPA-ER significantly stabilizes serum levels without marked peak-to-trough fluctuations, reduces dosing frequency and the possibility of dosing flexibility, which improves patient compliance, satisfaction and ultimately the quality of life.Although once-daily formulation is more convenient than multiple doses per day, potential subtherapeutic concentrations following delayed or missed doses should be concerned. Once-daily formulation is unable to improve therapeutic coverage cause, because it cannot maintain the effective drug concentration in biological fluids and tissues pharmacokinetically.A missed dose of once-daily formation usually poses a greater influence on the treatment due to the higher dose than that of multiple-dose formulation. Therefore, the risk of breakthrough seizure is higher in once-daily AED administration than that of twice-daily administration.VPA-ER is limited by the high daily dose owing to the lower bioavailability. It is reported that a mean increases in the daily dose of divalproex-ER at $12 \%(8-20 \%)$ is able to achieve an equivalent plasma exposure level to that of divalproex-DR.

Condition being studied: To our knowledge, comparison and conversion studies about VPA-ER and conventional VPA-DR are lacked. Small-sample studies analyzing the safety and efficacy of VPA-ER in different populations remain controversial.
Therefore, this study aims to systematically review the safety, efficacy and retention rate of VPA-ER and VPA-DR by metaanalysis.

\section{METHODS}

Search strategy: We will search articles in three electronic database including PubMed, EMBASE and Cochrane Library without language limitation. All the publications until 30 October, 2021 will be searched without any restriction of countries or article type. Reference list of all selected articles will independently screened to identify additional studies left out in the initial search.

Participant or population: Inclusion criteria for our studies : Any patients receiving VPA-ER without disease limitations.

Intervention: Extended-release formulation of VPA.

Comparator: Delayed-release formulations of VPA.

Study designs to be included: Randomized controlled blind/double-blind, crossover, openlabeled trialsRandomized controlled trials (RCTs) will be included.

Eligibility criteria: Inclusion criteria: Randomized controlled blind/double-blind, crossover, openlabeled trials reporting the efficacy, safety, medicine compliance (e.g., reduction in epileptic seizure frequency) of VPA-ER and the control were included. Exclusion criteria: (1) Observational studies, cohort studies, reviews, case report, letters, communication, editorials, lectures, conference abstracts; (2) Unclearly defined divalproex-ER; (3) Literatures with missing or repeated data.

Information sources: Electronic databases ,Moreover, references in each eligible literature were manually reviewed to avoid missing data.

Main outcome(s): Efficacy assessment, such as at least $\mathbf{5 0} \%$ reduction in seizure frequency or complete seizure freedom. 
Additional outcome(s): Safety and retention rate assessment.

Data management: Two authors will independently extract data. Any disagreement will be resolved by discussion until consensus is reached or by consulting a third author.The following data will be extracted: author, year of publication, total number of people included in the study, mean age, original inclusion criteria, study period, doses of progesterone and time of application.

Quality assessment / Risk of bias analysis: The quality of included literatures was independently reviewed by two reviewers using the Cochrane handbooks.including the following 7 aspects: random sequence generation, allocation concealment, blinding of participants and personnel, blinding of assessors, incomplete outcome data and selective outcomes reporting.

Strategy of data synthesis: Stata 16.0 software was used for statistical analyses. A dichotomous analysis with the risk ratios (RRs) and 95\% confidence intervals (Cls) were calculated to compare differences between groups. The heterogeneity was assessed by the Cochran's $Q$ test and 12 test. If $\mathbf{P} 50 \%$, data were analyzed using a random-effects model; Otherwise, they were analyzed using a fixed-effects model. Two-tailed $\mathrm{P}<0.05$ was considered statistically significant.

Subgroup analysis: If necessary, subgroup analysis will be performed to evaluate the main trials demonstrating a substantial impact on the inter-study heterogeneity.

Sensitivity analysis: If necessary, a leaveone-out sensitivity analysis will be performed to evaluate the main trials demonstrating a substantial impact on the inter-study heterogeneity.

Language: None restriction.

Country(ies) involved: China.

Keywords: Divalproex; Extended release; Efficacy; Safety.
Contributions of each author:

Author 1 - Chen qi Zhang.

Email: zcq4869@163.com

Author 2 - Xueyang Bai.

Author 3 - Yong Wan.

Author 4 - Hongyan Li.

Author 5 - Hongbin Sun.

Email: shb001369x@163.com 